\title{
Decoding the sweet regulation of apoptosis: the role of glycosylation and galectins in apoptotic signaling pathways
}

\author{
Kamil Seyrek $^{1} \cdot$ Max Richter $^{1} \cdot$ Inna N. Lavrik ${ }^{1}$ \\ Received: 8 January 2019 / Revised: 2 February 2019 / Accepted: 25 February 2019 / Published online: 22 March 2019 \\ ( ) ADMC Associazione Differenziamento e Morte Cellulare 2019
}

\begin{abstract}
Glycosylation and glycan-binding proteins such as galectins play an important role in the control of cell death signaling. Strikingly, very little attention has been given so far to the understanding of the molecular details behind this key regulatory network. Glycans attached to the death receptors such as CD95 and TRAIL-Rs, either alone or in a complex with galectins, might promote or inhibit apoptotic signals. However, we have just started to decode the functions of galectins in the modulation of extrinsic and intrinsic apoptosis. In this work, we have discussed the current understanding of the glycosylation-galectin regulatory network in CD95- as well as TRAIL-R-induced apoptosis and therapeutic strategies based on targeting galectins in cancer.
\end{abstract}

\section{Facts}

- CD95 and TRAIL-R are glycosylated, which modulates their sensitivity toward apoptosis induction.

- Galectins can regulate apoptosis by interacting with death receptors (DRs) and cell surface glycoproteins.

- Intracellular galectins modulate apoptosis via binding to glycoproteins as well as non-glycosylated proteins.

- O-glycosylation plays a key role for TRAIL-R2 system, while for TRAIL-R1 and CD95, N-glycosylation seems to be of major importance.

- Galectins can be used as potential targets for cancer therapy.

\section{Open questions}

- Identification of putative interactions between different types of galectins and DRs.

\begin{tabular}{l}
\hline Edited by S. Nagata \\
\hline$\square$ Inna N. Lavrik \\
inna.lavrik@med.ovgu.de \\
$1 \quad$ Translational Inflammation Research, Institute of Experimental \\
Internal Medicine, Medical Faculty, Otto von Guericke University, \\
Pfälzer Platz 2, 39106 Magdeburg, Germany
\end{tabular}

- Structural analysis of glycosylated DR complexes.

- Molecular mechanisms of galectin action in apoptosis and other types of cell death.

- Influence of proapoptotic and antiapoptotic galectins on DR-mediated cell death.

- Development of new combinatorial therapies based on targeting galectins and DR pathways.

\section{Introduction}

Apoptosis is a genetically encoded process and common to all multicellular organisms. There are two key ways of apoptosis induction: the death receptor (DR)-mediated or extrinsic pathway and the mitochondrial or intrinsic pathway $[1,2]$. The extrinsic cell death pathway can function independently of mitochondria and is triggered by stimulation of the DR family.

DRs belong to the tumor necrosis factor receptor (TNF-R) superfamily and are characterized by the presence of an intracellular death domain (DD) that is involved in the transduction of the death signal [3]. Six members of the DR family have been characterized to date: TNF-R1, CD95 (APO-1/FAS), DR3, TNF-related apoptosis-inducing ligand receptor 1 (TRAIL-R1)/DR4, TRAIL-R2/DR5, and DR6 [4]. The activation of CD95 or TRAIL-R1/2 with the cognate ligands or agonistic antibodies results in the formation of the death-inducing signaling complex (DISC) at the cellular membrane [5]. 


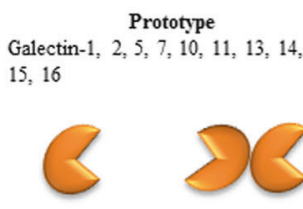
Dimer

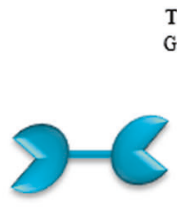

Monomer
Tandem-repeat type Galectin- $4,6,8,9,12$

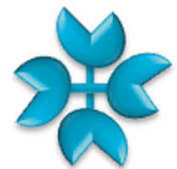

Dimer

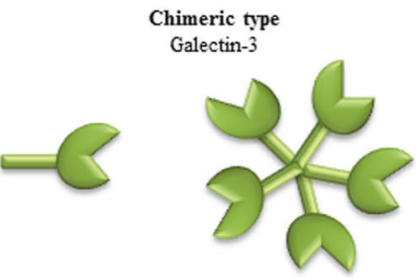

Monomer
Oligomer

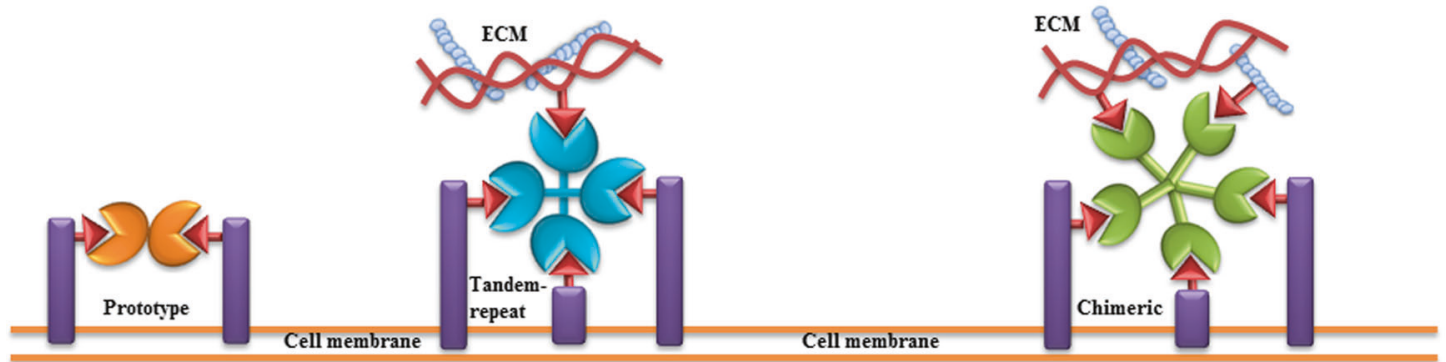

Fig. 1 Classification of galectins.According to the number and arrangement of the carbohydrate recognition domains (CRDs), galectin family members are classified into three main types: proto, chimera, and tandem-repeat. Some galectins can self-associate into dimers or oligomers. The consensus sequence, which corresponds to the CRD, consists of $\sim 130$ amino acids. Via their CRDs, galectins can interact with poly- $N$-acetyllactosamine (Gal- $\beta(1-4)$-GlcNAc, LacNAc)-based

DISC consists of oligomerized receptors, the adaptor protein Fas-associated protein with DD (FADD), initiator zymogens procaspase- 8 and -10 , and the cellular FLICE inhibitory protein (c-FLIP) [6, 7]. The protein-protein interactions in this complex are mediated via homotypic interactions, namely, the DD of the receptor interacts with the DD of FADD, the death effector domain (DED) of FADD interacts with DEDs of procaspase-8 and -10 and $c-\mathrm{FLIP}_{\mathrm{L} / \mathrm{S} / \mathrm{R}}$. Furthermore, via DED interactions, DED filaments are formed that serve as a platform for procaspase-8 activation [8-10]. Activated caspase- 8 induces the activation of downstream effector caspases-3 and -7 resulting in cleavage of several cellular substrates, such as poly ADP ribose polymerase [5].

Biological information coded in carbohydrate structures is translated by sugar-binding proteins called lectins. Animal lectins have been attracting attention for their roles in the extrinsic and intrinsic apoptotic pathways in several diseases [11]. Galectins, a family of galactose-binding lectins known as $\mathrm{Ca}^{2+}$-independent $\mathrm{S}$-type animal lectins, are widely distributed in many types of mammalian cells [11].

In spite of extensive investigations focused on CD95 and other DR signaling pathways, very few studies, however, have investigated the effects of posttranslational modifications such as glycosylation on DR function. Glycosylation of DRs in mammalian systems is likely to play a more prominent role than initially anticipated and will be considered below. carbohydrates present in proteins, lipids, or other molecules. "One-CRD" galectins usually exist as dimers, whereas Gal-3 forms upon binding to Gal- $\beta(1-4)-G l c N A c$ structures on cell surface and extracellular matrix. Tandem-repeat-type galectins comprise two homologous CRDs, separated by a short linker of up to 70 amino acids. Figure is modified from [100]

\section{Galectins as receptors of glycosylated proteins}

Galectins are highly conserved family of lectin molecules. Sixteen mammalian galectins have been identified to date [12]. According to the number and arrangement of the carbohydrate recognition domains (CRDs), galectins are classified into three main types: prototype (galectin-1, $-2,-5,-7$, $-10,-11,-13,-14,-15,-16)$, chimera (galectin-3 (Gal-3)), and tandem-repeat (galectin-4, -6, -8, -9, -12) [13-15] (Fig. 1). All three types of galectins have a well-defined CRD with a highly conserved amino acid sequence and a $\beta$-sandwich structure. The consensus sequence that corresponds to the CRD consists of $\sim 130$ amino acids. Galectins can interact via their CRDs with LacNAc-based $\mathrm{N}$-and O-linked glycans present in proteins, lipids, or other molecules [16-19]. Prototypical galectins possess one CRD, while the tandemrepeat-type galectins comprise two homologous CRDs, separated by a short linker of up to 70 amino acids [20, 21]. Gal-3 contains a collagen-like sequence linking the short 12 amino acid N-terminal domain to the CRD [21]. Galectins exist either in bivalent or multivalent form depending on their carbohydrate-binding activities [22]. They mostly form homodimers through hydrophobic or electrostatic interactions [22]. Tandem-repeat-type galectins are reported to be monomeric; however, some galectins of this class, like Gal-9, self-associate and form homodimers [23] (Fig. 1). Gal-3 is the only known chimera-type galectin that might assemble into multimers [24]. 
Galectins play different roles in various biological processes, such as cell cycle regulation, cell growth, and apoptosis [25-29]. There is an accumulated evidence that galectins modulate signal transduction by binding to intracellular ligands and participating in intracellular signaling pathways [30, 31]. However, the localization of galectins is not restricted to the intracellular compartments [22]. Several secreted galectins such as Gal-1, Gal-3, Gal-4, Gal-8, and Gal-9 are localized on the cell membrane and in the extracellular matrix (ECM) [21, 32]. Despite the lack of the signal sequence for a classical secretion, galectins are suggested to be secreted by a non-classical pathway. The latter is likely mediated via an exosome-mediated secretory route $[33,34]$. Extracellular galectins can interact with cell surface glycoproteins, harboring the LacNAc disaccharide structure. Through the binding to poly-LacNAc structures, galectins can generate a galectin-glycan lattice, which is able to regulate cellular signal transduction [33-35].

In the extracellular space, galectin homodimers can link several membrane receptors facilitating cell signaling and cell-cell interactions [21, 22]. For example, interactions of Gal-1 with N-and/or O-glycans on activated T cells lead to clustering and aggregation of CD45 with CD3 and CD7 with CD43 on $\mathrm{T}$ cells, which are crucial for signaling in $\mathrm{T}$ cells $[22,31,36]$.

\section{Modulation of CD95-induced apoptosis by glycosylation}

The extrinsic apoptotic pathway is initiated by stimulation of DRs such as CD95 and TRAIL-R1/2 and the subsequent formation of the DISC and DED filaments [1, 8-10, 37, 38] (Fig. 2). Upon recruitment to the DISC, procaspase- 8 is activated at the DED filaments (Fig. 2), which results in the formation of the active caspase- 8 heterotetramer $\mathrm{p} 10_{2}-\mathrm{p} 18_{2}$ and the propagation of the apoptotic signal [38, 39]. Two pathways of DR signaling have been described. Type I cells show a high level of DISC formation and caspase- 8 activation upon CD95 stimulation. In Type I cells, activated caspase- 8 directly leads to the activation of downstream effector caspase-3 and -7 [6]. In Type II cells, a low level of the DISC formation is observed and the apoptotic signal is amplified by the release of cytochrome $\mathrm{C}$ from the mitochondria through the cleavage of $\mathrm{BH} 3$ interacting-domain death agonist (Bid) to generate truncated Bid (tBid) and subsequent tBid-mediated release of cytochrome $\mathrm{C}$ from mitochondria. The release of cytochrome $\mathrm{C}$ from mitochondria results in apoptosome formation, followed by activation of procaspase-9, which in turn cleaves downstream, effector caspase-3 and -7 [40].

CD95 is one of the best studied DRs that harbors glycosylation sites both in the extracellular and intracellular domains [41-43]. N-glycosylation sites in the extracellular domain are located at the positions N118 and N136 (Fig. 2), whereas a potential site of $\mathrm{N}$-glycosylation resides at the position N223 of the intracellular domain [42]. Furthermore, an O-glycosylation site in the intracellular domain of CD95 has been predicted [42]. The glycosylation site at $\mathrm{N} 118$, but not at N136, is well conserved. Interestingly, the structural modeling has predicted that, due to its close proximity to CD95 ligand (CD95L)-binding site, N136 forms hydrogen bonds with residues 200-204 of CD95L. Thus glycosylation at N136 might contribute to the CD95L binding more than glycosylation at N118, which is located more distal from the CD95-CD95L interface [42].

The role of CD95 glycosylation in regulation of CD95 signaling is still controversially discussed. One line of suggestions is that N-glycosylation of CD95 is crucially required for the binding of CD95L [42, 44]. Indeed, considering the above-mentioned close proximity of N136 and N118 to CD95L-binding site, the glycosylation of these residues might either be directly involved in interactions with CD95L or drastically change the conformation of this region. This would modulate the binding of $\mathrm{CD} 95 \mathrm{~L}$ and thereby directly influence CD95-mediated response. However, in contrast to this line of evidence, it was also reported that N-deglycosylation of CD95, does not fully block the recruitment of FADD to the DISC and results only in a slight decrease of procaspase- 8 activation at the DISC [42]. The latter findings might indicate that $\mathrm{N}$-glycosylation of CD95 is required only for fine-tuning of CD95/CD95L interactions and is not absolutely necessary for CD95L binding to CD95. Supporting this suggestion is the evidence that Type I and Type II cells apparently have a different glycosylation pattern of CD95 [42]. Deglycosylation experiments performed on these two cell types indicate differential glycosylation pattern of CD95 between Type I and Type II cells [42], which precludes the universal role of $\mathrm{N}$-glycosylation of CD95 in maintaining CD95L binding. Hence, the influence of the extracellular N-glycosylation on CD95/CD95L interaction is one of the key questions that has to be addressed in future research. The important role in these studies should belong to the state-of-the-art structural approaches like nuclear magnetic resonance and X-ray analysis.

Yet another line of evidence for the role of CD95 glycosylation is based on the reports that CD95-associated $\mathrm{N}$-glycans contribute to the crosslinking of the adjacent DISCs and thereby facilitate procaspase- 8 oligomerization between adjacent receptor complexes [45]. In line with these data is the study by Charlier et al. which has reported that, in the case of aberrant glycosylation of CD95 in Jurkat cells, oligomerization of CD95 fails in response to CD95L [45]. These reports strongly suggest that an extracellular $\mathrm{N}$-glycosylation of CD95 has a major influence on the 


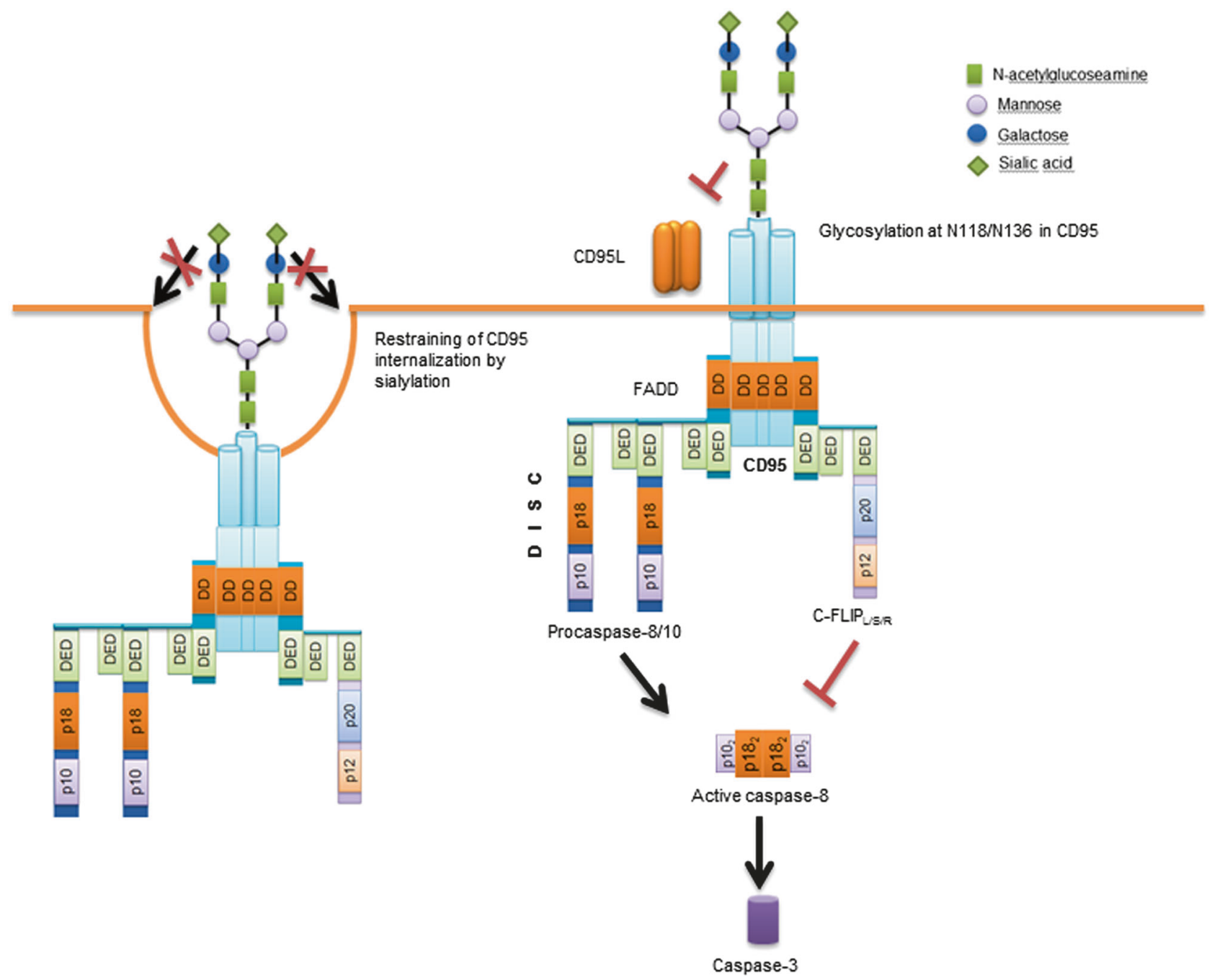

Fig. 2 Glycosylation network of CD95 and CD95-mediated apoptosis. Triggering CD95 by CD95L or agonistic antibodies induces its oligomerization and the subsequent formation of the death-inducing signaling complex (DISC). At the DISC, procaspase-8 undergoes dimerization in the death effector domain filaments that is followed by

CD95 oligomerization, and the latter has been several times highlighted as a key event in the initiation of CD95-mediated signal [46].

The other important factor, which has to be considered with respect to the role of $\mathrm{N}$-glycosylation in CD95 signaling is the role of sialylation. CD95 was reported to be sialylated on the $\mathrm{N}$-linked oligosaccharide residues (Fig. 2) [41, 47]. In many cell types, sialic acid residues on CD95 can account for up to $8 \mathrm{kDa}$ increase of its molecular weight [41]. Mentioned above differences between CD95 glycosylation pattern in Type I vs. Type II cells might be also directly associated with the differences in the sialylation of the receptor between distinct cell types. Several studies have suggested that CD95 apoptotic activity might be inhibited by sialylation. Swindall et al. reported that $\alpha 2-6$ sialylation of CD95 does not interfere with the activation of caspase- 8 . The mature caspase- 8 , consisting of two p18 and two p10 subunits, cleaves and activates downstream effector caspases, including caspase-3. CD95 is N-glycosylated in the extracellular domain, which can modulate the function of CD95 by several mechanisms discussed in the text

binding of the agonist but rather inhibits formation of the DISC and also restrains CD95 internalization [43]. In line with these reports, other studies have shown that in various human $\mathrm{B}$ and $\mathrm{T}$ cell lines desialylation of CD95 using Vibrio cholera neuraminidase increases the sensitivity of these cells toward CD95-induced apoptosis [41, 42, 47]. Hence, the degree of sialylation of CD95 provides yet another checkpoint in the regulation of CD95-mediated apoptosis. Nevertheless, the detailed mechanisms of sialylation control at CD95 still have to be uncovered. In particular important will be to get the structural information on the structure of sialylated CD95 in a complex with CD95L as well as to obtain a quantitative information upon the degree of sialylation that serve as "a sensitivity threshold" toward CD95-induced apoptosis. The latter can be achieved through the cutting edge technologies of 
quantitative proteomics in combination with the glycoproteome analysis [48].

\section{Modulation of TRAIL-induced signaling by glycosylation}

In the regulation of TRAIL signaling, glycosylation of TRAIL receptors also plays an important role [49]. In particular, O-glycosylation of human TRAIL-R2, has been reported to be crucially required for receptor clustering, DISC formation, caspase- 8 recruitment, and downstream signaling in the TRAIL/TRAIL-R2-mediated apoptosis [49, 50]. TRAIL-R2 is O-glycosylated on two stretches of serines and threonines within and around the cysteine-rich domains 2 and 3. Interestingly, it has been suggested that O-glycosylation does not alter TRAIL-binding affinity; rather, it promotes ligand-induced receptor clustering [50]. The latter is in line with the above-mentioned reports on the role of CD95 glycosylation in receptor oligomerization, which allows to suggest that the extracellular glycosylation might be essential for DR oligomerization and subsequent signal transduction.

Interestingly, no extracellular N-linked glycosylation sites were reported for TRAIL-R2. However, TRAIL-R1 has an N-glycosylation site at N156, which is located within the first cysteine-rich domain of the receptor. Moreover, recently it has been shown that N-glycosylation at N156 of human TRAIL-R1 enhances its ability to trigger apoptosis [49]. Similar to the role of O-glycosylation in TRAIL-R2 signaling, N-glycosylation of TRAIL-R1 is suggested to be required for the DISC formation and procaspase- 8 activation [49]. An interesting question remains why O-glycosylation plays a key role for the TRAIL-R2 system, while for signaling of TRAIL-R1 and CD95, $\mathrm{N}$-glycosylation seems to play a crucial role and whether there are some structural differences in extracellular domains of these three receptors that dictate these properties. This question should be addressed in future studies.

Contrary to the above-mentioned investigations, Estornes et al. reported in a comprehensive study that, in mouse embryonic fibroblasts (MEFs), which were completely resistant to TRAIL, the removal of N-linked glycans of TRAIL-R by tunicamycin treatment sensitizes the cells to TRAIL-induced apoptosis [51]. Deglycosylation-dependent sensitization to TRAIL-induced apoptosis was not only limited to MEFs but was also reported in the same study for mouse HT-22 hippocampal neuronal cells and 3T3 fibroblasts [51]. Moreover, a non-N-glycosylated form of mouse TRAIL-R has been shown to present at the plasma membrane and possess a higher binding capacity for TRAIL in vitro assays [51]. One possible explanation of this discrepancy could be the different cell types used in this study that asks for the cell-type-specific analysis of DR glycosylation in the future investigations. Furthermore, the above-mentioned study from Shatnyeva et al. [42] also did not report drastic changes in the DISC formation and a transmission of CD95 signal upon deglycosylation of CD95, which would support the hypothesis that in some cell types DR glycosylation is not crucially important for ligand binding and apoptosis induction and is only required for the fine-tuning of the apoptosis signal.

Finally, it has to be noted that the analysis of glycosylation is rather complicated because many of the glycosylation inhibitors possess a cytotoxicity, while introduction of DR glycosylation mutants switches the cellular systems towards overexpression of DRs, which also might cause side effects. In this regard, future studies using cutting edge structural and glycoproteomics approaches are of high relevance as they would allow to delineate the proapoptotic or antiapoptotic roles of DR extracellular glycosylation and solve the contradictions in the literature.

Yet another important question to address is whether glycosylation of the DRs has an influence on the induction of the antiapoptotic pathways such as nuclear factor-kB or mitogen-activated protein kinase (MAPK) activation as well as induction of other cell death programs, including necroptosis. Indeed, there is a growing interest to the induction of these pathways via DRs, accordingly, the role of glycosylation in this signal transduction has to be undoubtedly addressed in future studies [5, 52].

\section{The role of galectin-3 and other galectins in CD95/TRAIL-R-mediated cell death}

Gal-3 binds to both CD95 and TRAIL-Rs at their extracellular domains through LacNAc interactions. Strikingly, this binding seems to have completely different effects on TRAIL-R vs. CD95 signaling. Gal-3 has been reported to crosslink TRAIL-Rs on human colon cancer cells by trapping them in a so-called nano-cluster lattice, which in turn results in blocking the DISC formation [53]. In particular, a cell surface Gal-3 on metastatic colon cancer cells has been shown to form heterodimeric complexes with glycosylated TRAIL-R1 and TRAIL-R2, thus blocking efficient DISC formation and preventing the apoptotic signal [53]. Depletion of Gal-3 using Gal-3 short hairpinRNAs did not influence the amount of cell surface TRAIL-R1 or TRAIL-R2 but restored the sensitivity of TRAIL-resistant colon cancer cells. Furthermore, glycosylation inhibitors such as swainsonine, tunicamycin, or benzyl-alpha- $N$ Acetyl-galactosamine re-sensitized TRAIL-resistant cells, which likely was due to the inhibition of the interaction between Gal-3 and TRAIL-Rs [53]. In line with antiapoptotic effects of Gal-3 on colon cancer cells, there were reports that in human bladder carcinoma cancer cells 
knockdown of Gal-3 can promote TRAIL-induced apoptosis $[54,55]$.

There are also few studies concerning the direct interactions between Gal-3 and CD95; however, in contrast to TRAIL pathways, Gal-3 seems to play a proapoptotic role in CD95 signaling. Fukumori et al. revealed that Gal-3 is a key factor defining the difference between CD95-induced apoptotic pathway in Type I and Type II cells [56]. In particular, Gal-3 has been reported to induce DISC formation via a direct binding to CD95 only in Type I and not in Type II cells. These findings may explain the differences between Type I and Type II cells. Accordingly, the different roles of Gal-3 on TRAIL-Rs vs. CD95 signaling might be linked to the differential pattern of glycosylation of the extracellular regions of CD95 vs. TRAIL-Rs. This would result in the distinct architecture of receptor complexes upon Gal-3 binding and accordingly lead to the different signaling outcome. Understanding this question might be also obtained via a detailed structural analysis of the DR structure.

Gal-1 has been also reported to interact with extracellular domain of CD95 in Jurkat, CEM, and resting T cells. Interactions of Gal-1 with CD95 have been reported to induce CD95 clustering, caspase-8 recruitment, and activation of initiator caspases leading to CD95L-mediated apoptosis $[44,57,58]$. Thus it seems that Gal-1 similar to Gal-3 promotes CD95-induced apoptosis, thus demonstrating its proapoptotic role in CD95 signaling.

Similar to proapoptotic effects of Gal-3 and Gal-1 on CD95 signaling, Gal-9 binds to the extracellular domains of DRs through LacNAc units and induces apoptosis in several cell types [59-62]. For example, in human T leukemia cells Gal-9 administration increases caspase-8 activation [63]. Abrogation of this process by lactose, an effective inhibitor of Gal-9, indicates that Gal-9 implements apoptosis through DR-mediated signaling [63, 64].

Taken together, both proapoptotic and antiapoptotic effects of galectins on DR signaling pathway have been reported so far. The further insight into this question should be provided by the detailed analysis of glycostructure of the extracellular domains of CD95 vs. TRAIL-Rs as well as the structure of these regions upon binding of galectins. Unraveling these differences should uncover new important insights into the mechanisms of galectins action. The other important highlight will be to address the role of galectins in the non-apoptotic DR-mediated signaling pathways, which has attracted a lot of attention recently.

\section{Further functions of galectins in apoptosis and other signaling pathways}

Signaling pathways modulated by galectins are not restricted to DR signaling pathways and their extracellular roles as described in the previous chapter. For example, intracellular Gal-1 has been reported to block the cell cycle in the late $\mathrm{S}$ phase by altering the expression of transcription factor E2F1 [65]. Gal-1-mediated blockage of cell cycle in $\mathrm{S}$ phase increases transcription factor E2F1 levels leading to the activation of apoptosis in tumor cells, whereas in normal cells Gal-1 has been reported only to block growth arrest $[65,66]$. Furthermore, it has been shown that intracellular Gal-1 induces apoptosis in human $\mathrm{T}$ cells in a caspaseindependent manner by inducing the translocation of endonuclease $\mathrm{G}$ from mitochondria to nuclei [67]. Through a prenyl-binding pocket, Gal-1 is able to selectively bind GTP-bound HRAS. This coupling alters the orientation of the HRAS globular domain on the cell membrane, leading to the lateral segregation of HRAS and promotion of the MAPK signaling [68-70].

Upregulation of Gal-2 in activated $\mathrm{T}$ cells has been reported to be linked to the increase of Bax and downregulation of $\mathrm{Bcl}-2$ levels [71], favoring proapoptotic functionality in the course of the downmodulation of immune response. Interestingly, Gal-2 interacts preferentially with CD29. Accordingly, co-stimulation of CD29 may provide an additional signal required for apoptosis of activated $\mathrm{T}$ cells.

Proapoptotic effect of Gal-3 is also not restricted to DRs. Gal-3 has been reported to trigger apoptosis via binding to several receptors other than DRs, such as CD7, CD29, CD45, CD71, and CD98 [56, 72]. However, the underlying molecular mechanisms still have to be unraveled. For instance, the secreted Gal-3 has been reported to interact with the CD29/CD7 complex and mediate the activation of apoptosis in T cell lines [73] (Fig. 3). Interestingly, Gal-3mediated CD29-induced apoptosis requires recruitment of caspase- 8 to the membrane [74]. The latter might indicate potential crosstalk with DR pathways that has to be addressed in future studies. Moreover, extracellular Gal-3 mediates endocytosis of CD29 via a caveolae-like signaling pathway [75]. Additionally, Gal-3 interacts with CD98 on the cell membrane and induces apoptosis through $\mathrm{Ca}^{2+}$ influx pathway [75]. Similar to Gal-1, Gal-3 is involved in RAS pathway by binding to the farnesyl group of GTPbound KRAS and acting as a scaffold for KRAS anchoring to the plasma membrane [70]. Moreover, multivalent Gal-3 can generate galectin-glycan lattices not only via crosslinking of TRAIL-Rs but also via binding to several cell surface glycoproteins, such as growth factor receptors epidermal growth factor receptor (EGFR), K-Ras, vascular endothelial growth factor (VEGF) and basic fibroblast growth factor, and $\mathrm{T}$ cell receptor and ECM proteins, like fibronectin $[54,76]$.

One of the extraordinary features of Gal-3 is that Gal-3 displays a significant sequence similarity with antiapoptotic protein Bcl-2. There is about $28 \%$ identity and $48 \%$ similarity 


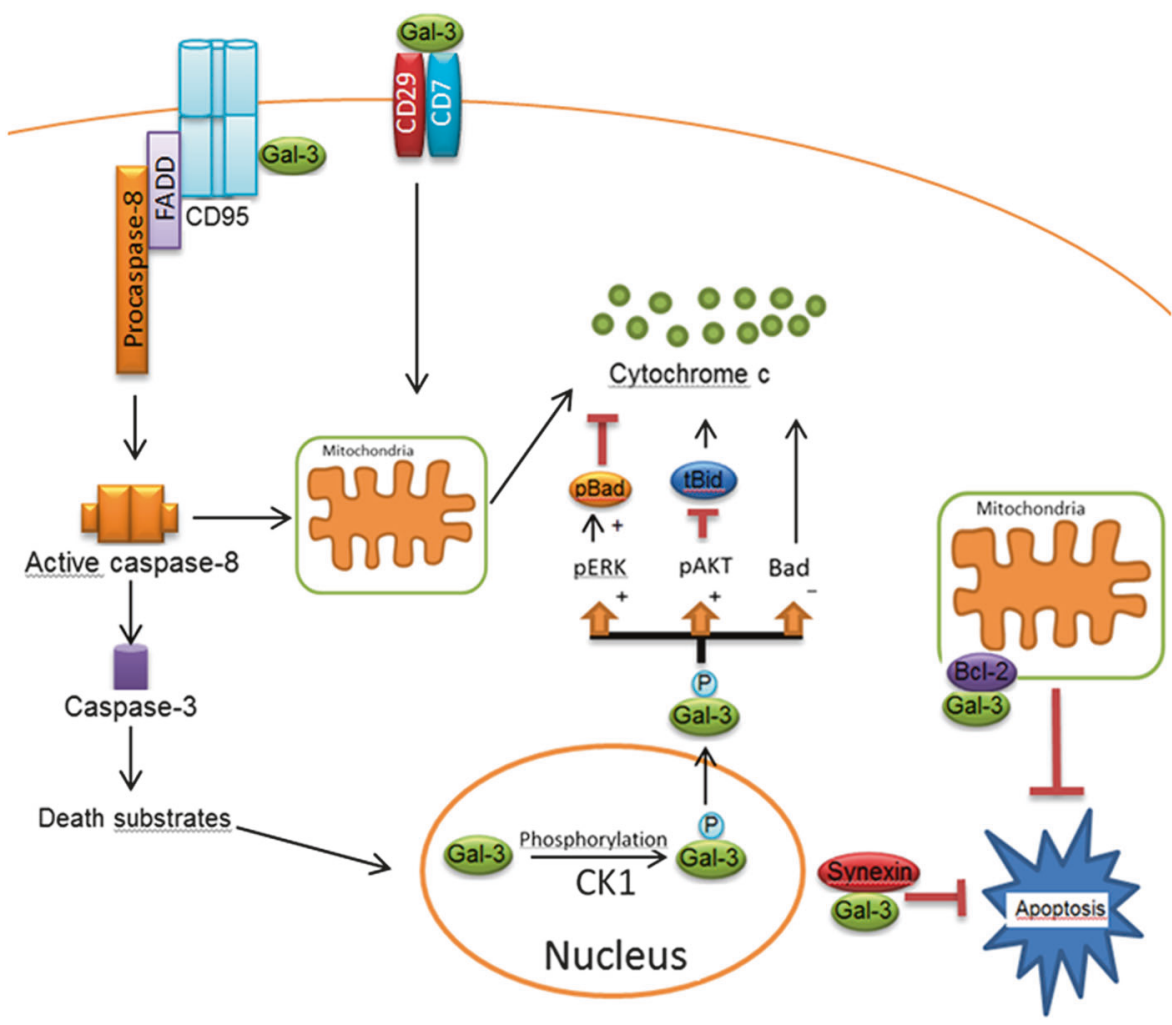

Fig. 3 Apoptotic network of Gal-3 in extrinsic and intrinsic pathways. Gal-3 comprises a serine phosphorylation site, which is involved in its translocation into the cytosol. Following the phosphorylation of Gal-3 by Casein Kinase 1 (CK1), Gal-3 translocates from the nucleus to the cytoplasm. The phosphorylated form of Gal-3(pGal-3) in the cytoplasm induces Bad phosphorylation via Erk pathway. pGal-3 promotes Akt activation, which blocks cleavage of Bid into tBid preventing mitochondrial outer membrane polarization. Gal-3 also attenuates

between these two protein sequences [40]. Moreover, both Gal-3 and Bcl-2 have a tendency to self-associate and form homodimers [77, 78], but cytosolic Gal-3 can also form heterodimers with Bcl-2. The CRD of Gal-3 comprises 110-130 amino acids with a unique aspartate-tryptophanglycine-arginine (NWGR) motif, which is essential for Gal-3/ Bax heterodimerization [79]. Interestingly, the NWGR motif in $\mathrm{Bcl}-2$ is also essential for $\mathrm{Bcl}-2 / \mathrm{Bax}$ heterodimerization. Accordingly, Gal-3 CRD binds to the BH1 domain of Bax instead of Bcl-2 $\mathrm{BH} 3$, which prevents Bax oligomerization similar to the mechanisms that occur in the course of Bcl-2/ Bax heterodimerization. Hence, the similar mechanisms are responsible for the antiapoptotic activity of both intracellular Gal-3 and Bcl-2 [79]. Intracellular Gal-3 has the ability to interact with Bax in breast, bladder, and prostate cancer cells $[80,81]$. Thus the reported role of Gal-3 in intrinsic pathway regulation seems not to solely involve its glycan-binding properties.

Moreover, Gal-3 overexpression in Jurkat cells endows them more resistant to apoptosis induced by staurosporine or anti-CD95 antibodies [31]. Furthermore, Gal-3 has been cytochrome $\mathrm{C}$ release from the mitochondria by decreasing Bad expression. Synexin is required for Gal-3 translocation from the nucleus into the cytoplasm. In Type I cells, Gal-3 has been reported to interact with CD95, which results in high amounts of death-inducing signaling complex and active caspase-8. Extracellular Gal-3 binds to the CD29/CD7 complex, which triggers the activation of an intracellular apoptotic signaling cascade followed by cytochrome $\mathrm{C}$ release from mitochondria. Figure is modified from [32]

reported to translocate to the perinuclear membrane upon several apoptotic stimuli. Confocal microscopy and biochemical analysis revealed that Gal-3 is accumulated in the mitochondria and prevents cytochrome $\mathrm{C}$ release and subsequent apoptosis [82-84]. Hence, future studies have to identify the exact molecular mechanisms of the intracellular Gal-3 action at the mitochondria and delineate how Gal-3 triggers multiple apoptosis pathways.

The activity of Gal-3 is strictly controlled by posttranslational modifications. Gal-3 is phosphorylated at Ser6. Phosphorylation at Ser6 is mediated by Casein kinase I, and interestingly, it was reported that only the phosphorylated form of Gal-3 exhibits antiapoptotic activity [26, 55]. Strikingly, the translocation of Gal-3 from the nucleus to perinuclear mitochondrial membranes requires phosphorylation of this protein. However, a phosphorylated wildtype Gal-3 translocates from the nucleus to the cytoplasm and was reported to play an antiapoptotic role in DNA damage-induced apoptosis $[55,83]$. In addition, the phosphorylated form of Gal-3 has been reported to induce MAPK pathway and promote Akt activation resulting in 
inhibition of apoptosis by blocking cleavage of Bid into tBid [79] (Fig. 3). Thus phosphorylation of Gal-3 provides yet another way of its activity regulation and has to be investigated in future studies.

Gal-4 has the ability to interact with CD3 and CD4 in T cells. Gal-4 interaction with CD4 induces interleukin (IL)6 production, whereas binding to $\mathrm{CD} 3$ promotes $\mathrm{T}$ cell apoptosis through calpain-mediated pathway [44].

In colon carcinoma cells, Gal-7 directly interacts with Bcl2 in a carbohydrate-independent manner and promotes cytochrome $\mathrm{C}$ and second mitochondria activator of caspases (Smac)/direct IAP binding protein with low pI release [85]. In keratinocytes, increased levels of Gal-7 caused by ultraviolet B irradiation stabilize p53 and induce apoptosis. Proapoptotic feature of Gal-7 is thought not to be due to the interactions with cell surface glycoproteins. In fact, Gal-7 is present in the nuclei and cytoplasm of certain cell types and intracellular expression of Gal-7 enhances caspase-3 activity [86].

Gal-8 can promote apoptosis also by interacting with cell surface glycoproteins other than DRs, such as CD44. It is revealed that interaction of Gal-8 with CD44 induces apoptosis in joint inflammatory cells [87]. It has also been shown that through specific integrins, such as integrin $\alpha 1 \beta 1, \alpha 3 \beta 1$ and $\alpha 5 \beta 1$, Gal-8 induces apoptosis in Jurkat T cells as well as in human peripheral blood mononuclear cells by promoting extracellular signal-regulated kinase 1/2 (ERK1/2) activation. This leads to CD95L and IL-2 expression and subsequently caspase-mediated apoptosis in Jurkat T cells [88].

Gal-9-induced apoptosis is not restricted to DR-mediated pathway, rather Gal-9 induces apoptosis, for example, in MOLT4 cells through the $\mathrm{Ca}^{2+}$-calpain-caspase-1 pathway $[89,90]$. Interaction of Gal-9 with cell surface molecule Tim-3 on T helper type 1 cells causes apoptosis via intracellular calcium flux and cell aggregation [60]. Furthermore, Gal-9 triggers apoptosis in chronic myelogenous leukemia cells and myeloma cells through c-Jun N-terminal kinase and p38 MAPK pathways [60, 61].

Gal-12 plays an important role in the regulation of cell cycle and induces apoptosis in different cell types such as adipocytes and COS-1 cells [12, 91]. A strong downregulation in Gal-12 expression was observed in acute myeloid leukemia. Moreover, these patients have lower survival rate than the ones with the higher Gal-12 levels [12, 92].

Some signaling pathways in which galectins are involved are revealed. Although the exact molecular mechanisms of galectins action in the regulation of apoptosis remains elusive, galectins have generated significant interest in the modulation of apoptosis for cancer therapy. Furthermore, several cells can express more than one galectin concomitantly, and therefore for a better galectin-based therapeutic benefit, it is necessary to have a more thorough understanding of galectin-mediated apoptotic signaling pathway in different types of cancer cells. In this regard, several issues such as different functions exerted by the same galectin within different environmental contexts, transmission of death signals by galectins, and balance between intracellular and extracellular localization of galectins in apoptotic cells remain to be addressed.

\section{Galectins as potential therapeutic targets in cancer}

Targeting galectins, as highlighted above, presents a very attractive strategy in cancer therapy. There are several studies aiming to re-sensitize the tumor cells to apoptotic signaling pathway by targeting galectins. In particular, various galectin antagonists, such as $O$-galactosyl aldoximes [93], 3-(1,2,3-triazol-1-yl)-1-thio-galactosides [94], and phenyl thio- $\beta$-D-galactopyranosides [95] have been developed. The major principle of the rational design of these inhibitors is to block interactions between galectins and their binding partners.

Gal-1 is highly upregulated in tumor-activated endothelial cells and was reported to support tumor growth [96]. In particular, this lectin has been reported to induce apoptosis of CD4+ and CD8+ lymphocytes, preventing, at least partly, the tumor from the immune cell recognition, and facilitate tumor cell-endothelial cell interaction, promoting metastasis formation [97]. Gal-1 promotes angiogenesis through interaction with the VEGF co-receptor neuropilin-1 and Gal-1 knockdown in cancer cells reduces tumor angiogenesis by inhibiting endothelial cell migration and proliferation [98, 99]. Subsequently, a Gal-1 antagonist anginex binds specifically to Gal-1 and attenuates tumor angiogenesis and tumor growth [96, 97].

The other Gal-1 antagonist, galactomannan GM-CT-01 (DAVANAT $^{\circledR}$ ), increases the antitumor activity of 5fluorouracil in mice with colon tumor as well as in the other cancer types, such as lymphoma, breast cancer, and melanoma [100]. It is still not known exactly how DAVANAT acts as an anticancer agent. Inhibition of Gal-1 and Gal-3 by DAVANAT may prevent their binding to proapoptotic proteins that in turn induces apoptosis of cancer cells. The targets of Gal-1 and Gal-3 in cancer cells still have to be unraveled.

Ras proteins regulate signal transduction pathways controlling cell growth, differentiation, and survival. Ras genes, frequently mutated in tumors, promote malignant transformation. Ras transformation requires membrane anchorage and anchoring of HRAS to the plasma membrane is crucial step of tumor transformation leading to the sustained activation of Raf-1/MEK-1/ERK signaling pathway, which promotes tumor progression [100]. Gal-1 has membranetargeting signals and can selectively bind to GTP-bound HRAS and thereby enable its localization to the cell 


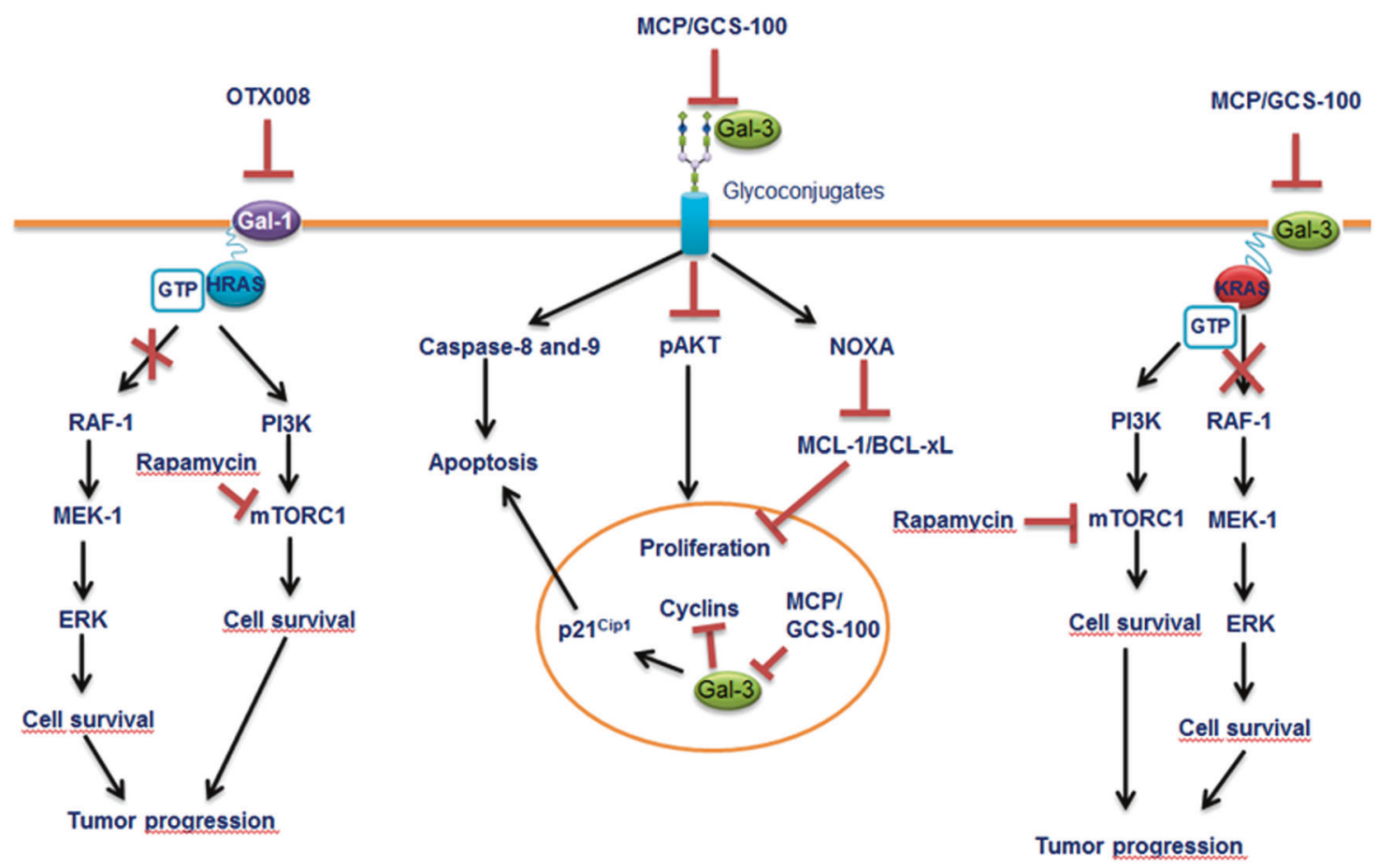

Fig. 4 Mechanisms of action of galectin inhibitors.Inhibition of Gal-1 by OTX008 results in HRAS mistargeting to the cell membrane, leading to the blockage of mitogen-activated protein kinase mitogenic pathway, whereas rapamycin decreases mammalian target of rapamycinsurvival signaling. Combination of OTX008 and rapamycin yields more effective treatment against tumor progression. Blockage of Gal-3 with MCP/GSC-100 activates caspase- 8 and -9 pathways and induces NOXA protein, leading to decrease in MCL-1 and BCL-XL.
Inhibition of nuclear Gal-3 induces cell-cycle inhibitor p21 Cip1 expression and blocks the expression of cyclins, leading to cell death. Inhibition of Gal-3 with MCP/GSC-100 reduces KRAS-activated tumor progression. The combined treatment using MCP/GCS-100 and rapamycin together, however, has revealed more promising results in the KRAS mutant tumor progression experimental models. Figure is modified from [101]

KRAS activation is associated with ERK but not phosphoinositide-3 kinase (PI3K) signaling [71]. Tumor cell activation based on Gal-3 activated KRAS can be inhibited, at least in part, by blocking of Gal-3 using MCP/ GCS-100 [101]. Furthermore, dual MAPK/PI3K inhibition using MCP/GCS-100 and rapamycin together, however, has revealed more promising results in the KRAS tumors. In Lewis lung carcinoma cells harboring one mutant $\mathrm{Kra}$ seallele encoding CA Kras ${ }^{\mathrm{G} 12 \mathrm{C}}$, MCP/GSC-100 selectively blocked the ERK pathway [101]. The co-treatment with MCP/GCS-100 and rapamycin of Lewis lung carcinoma cells also yielded stronger tumor-inhibitory effects [101].

Several studies suggest that galectins play an important role in tumor progression and metastasis by interfering with several mechanisms. Thus further development of galectin inhibitors opens new ways for treatment of cancer and thereby undoubtedly presents a very promising direction for future cancer research. Furthermore, considering the emerging role of galectins in the regulation of DR signaling, the combinatorial treatment with galectin inhibitors and targeting DR pathways might provide another important way of the sensitization of cancer cells toward cell death and has to be considered in future therapeutic approaches. for anchoring to the plasma membrane [101]. Gal-3-induced 


\section{Conclusion}

Here we have discussed DR glycosylation and the role of galectins in the modulation of apoptosis. Glycosylation modulates the function of DRs providing a very important level of apoptosis control. Interestingly, comparing glycosylation network of CD95 with TRAIL-R1/2, similar features can be found as well as some differences. All three receptors undergo glycosylation. Changes of their glycosylation status modulate the sensitivity of cells toward DRmediated apoptosis. Strikingly, O-glycosylation plays the key role for TRAIL-R2 system, while for TRAIL-R1 and CD95, N-glycosylation seems to have a major influence on the signaling outcome. Furthermore, there are rather contradictory reports on the role of DR glycosylation in the different cell types. These differences are probably tightly connected to the cell-type-specific glycostructure of DRs, which has to be addressed in future studies. Hence, glycans attached to the DRs, either alone or in a complex with glycan-binding proteins, can promote or restrain apoptotic signals. Among glycan-binding proteins that control apoptosis, the special attention belongs to galectins that are multifaceted proteins. Furthermore, we are only just now beginning to grasp the functions of galectins in the modulation of extrinsic and intrinsic apoptotic signaling pathways. However, the exact role of these interactions in the regulation of apoptosis remains elusive and major insights into detailed mechanisms of this regulatory network should be provided by the state-of-the-art structural analysis. In recent years, several reports have revealed that a correlation exists between expression of galectins and tumor progression. Moreover, depending on the type of galectin and/or galectin target protein, galectins can promote or impede apoptotic signaling pathways in cancer cells. Therefore, galectins have generated a significant interest in cancer therapy and further insight into their function would undoubtedly pave the new ways toward more efficient anticancer therapies.

Acknowledgements We acknowledge Volkswagen Foundation (VW 90315), Wilhelm Sander-Stiftung (2017.008.01), and Center of dynamic systems (CDS) funded by the EU-programme ERDF (European Regional Development Fund) and DFG (LA 2386) for supporting our work.

Competing interests The authors declare no competing interests.

Publisher's note: Springer Nature remains neutral with regard to jurisdictional claims in published maps and institutional affiliations.

\section{References}

1. Elmore S. Apoptosis: a review of programmed cell death. Toxicol Pathol. 2007;35:495-516.
2. Igney FH, Krammer PH. Death and anti-death: tumour resistance to apoptosis. Nat Rev Cancer. 2002;2:277-88.

3. Weber $\mathrm{CH}$, Vincenz $\mathrm{C}$. The death domain superfamily: a tale of two interfaces? Trends Biochem Sci. 2001;26:475-81.

4. Lavrik I, Golks A, Krammer PH. Death receptor signaling. J Cell Sci. 2005;118(Pt 2):265-7.

5. Krammer PH, Arnold R, Lavrik IN. Life and death in peripheral T cells. Nat Rev Immunol. 2007;7:532-42.

6. Scaffidi C, Schmitz I, Zha J, Korsmeyer SJ, Krammer PH, Peter ME. Differential modulation of apoptosis sensitivity in CD95 type I and type II cells. J Biol Chem. 1999;274:22532-8.

7. Sprick MR, Rieser E, Stahl H, Grosse-Wilde A, Weigand MA, Walczak H. Caspase-10 is recruited to and activated at the native TRAIL and CD95 death-inducing signalling complexes in a FADD-dependent manner but can not functionally substitute caspase-8. EMBO J. 2002;21:4520-30.

8. Fu TM, Li Y, Lu A, Li Z, Vajjhala PR, Cruz AC, et al. Cryo-EM structure of caspase-8 tandem DED filament reveals assembly and regulation mechanisms of the death-inducing signaling complex. Mol Cell. 2016;64:236-50.

9. Hughes MA, Powley IR, Jukes-Jones R, Horn S, Feoktistova M, Fairall L, et al. Co-operative and hierarchical binding of c-FLIP and caspase-8: a unified model defines how c-FLIP isoforms differentially control cell fate. Mol Cell. 2016;61:834-49.

10. Schleich K, Warnken U, Fricker N, Ozturk S, Richter P, Kammerer K, et al. Stoichiometry of the CD95 death-inducing signaling complex: experimental and modeling evidence for a death effector domain chain model. Mol Cell. 2012;47:306-19.

11. Liu Z, Zhang Q, Peng H, Zhang WZ. Animal lectins: potential antitumor therapeutic targets in apoptosis. Appl Biochem Biotechnol. 2012;168:629-37.

12. Wan L, Yang RY, Liu FT. Galectin-12 in cellular differentiation, apoptosis and polarization. Int J Mol Sci. 2018;19:E176.

13. Brinchmann MF, Patel DM, Iversen MH. The role of galectins as modulators of metabolism and inflammation. Mediat Inflamm. 2018;2018:9186940.

14. Bartolazzi A, Sciacchitano S, D'Alessandria C. Galectin-3: the impact on the clinical management of patients with thyroid nodules and future perspectives. Int J Mol Sci. 2018;19:E445.

15. Chou FC, Chen HY, Kuo CC, Sytwu HK. Role of galectins in tumors and in clinical immunotherapy. Int J Mol Sci. 2018;19: E430.

16. Advedissian T, Deshayes F, Viguier M. Galectin-7 in epithelial homeostasis and carcinomas. Int J Mol Sci. 2017;18:E2760.

17. Hirabayashi J, Hashidate T, Arata Y, Nishi N, Nakamura T, Hirashima $\mathrm{M}$, et al. Oligosaccharide specificity of galectins: a search by frontal affinity chromatography. Biochim Biophys Acta. 2002;1572:232-54.

18. Rabinovich GA, Toscano MA. Turning 'sweet' on immunity: galectin-glycan interactions in immune tolerance and inflammation. Nat Rev Immunol. 2009;9:338-52.

19. Stowell SR, Arthur CM, Mehta P, Slanina KA, Blixt O, Leffler $\mathrm{H}$, et al. Galectin-1, -2 , and -3 exhibit differential recognition of sialylated glycans and blood group antigens. J Biol Chem. 2008;283:10109-23.

20. de Boer RA, Yu L, van Veldhuisen DJ. Galectin-3 in cardiac remodeling and heart failure. Curr Heart Fail Rep. 2010;7:1-8.

21. Yang RY, Rabinovich GA, Liu FT. Galectins: structure, function and therapeutic potential. Expert Rev Mol Med. 2008;10:e17.

22. Dings RPM, Miller MC, Griffin RJ, Mayo KH. Galectins as molecular targets for therapeutic intervention. Int $\mathrm{J}$ Mol Sci. 2018;19:E905.

23. Nagae M, Nishi N, Murata T, Usui T, Nakamura T, Wakatsuki S, et al. Crystal structure of the galectin- $9 \mathrm{~N}$-terminal carbohydrate recognition domain from Mus musculus reveals the basic 
mechanism of carbohydrate recognition. J Biol Chem. 2006; 281:35884-93.

24. Ahmad N, Gabius HJ, Andre S, Kaltner H, Sabesan S, Roy R, et al. Galectin-3 precipitates as a pentamer with synthetic multivalent carbohydrates and forms heterogeneous cross-linked complexes. J Biol Chem. 2004;279:10841-7.

25. Barondes SH, Cooper DN, Gitt MA, Leffler H. Galectins. Structure and function of a large family of animal lectins. J Biol Chem. 1994;269:20807-10.

26. Fukumori T, Kanayama HO, Raz A. The role of galectin-3 in cancer drug resistance. Drug Resist Updat. 2007;10:101-8.

27. Liu FT, Patterson RJ, Wang JL. Intracellular functions of galectins. Biochim Biophys Acta. 2002;1572:263-73.

28. Nakahara S, Oka N, Raz A. On the role of galectin-3 in cancer apoptosis. Apoptosis. 2005;10:267-75.

29. Sacchettini JC, Baum LG, Brewer CF. Multivalent proteincarbohydrate interactions. A new paradigm for supermolecular assembly and signal transduction. Biochemistry. 2001;40:3009-15.

30. Ashery U, Yizhar O, Rotblat B, Elad-Sfadia G, Barkan B, Haklai $\mathrm{R}$, et al. Spatiotemporal organization of Ras signaling: rasosomes and the galectin switch. Cell Mol Neurobiol. 2006;26:471-95.

31. Yang RY, Hsu DK, Liu FT. Expression of galectin-3 modulates T-cell growth and apoptosis. Proc Natl Acad Sci USA. 1996;93:6737-42.

32. Storti P, Marchica V, Giuliani N. Role of galectins in multiple myeloma. Int J Mol Sci. 2017;18:E2740.

33. Brewer CF, Miceli MC, Baum LG. Clusters, bundles, arrays and lattices: novel mechanisms for lectin-saccharide-mediated cellular interactions. Curr Opin Struct Biol. 2002;12:616-23.

34. Nabi IR, Shankar J, Dennis JW. The galectin lattice at a glance. J Cell Sci. 2015;128:2213-9.

35. Rabinovich GA, Toscano MA, Jackson SS, Vasta GR. Functions of cell surface galectin-glycoprotein lattices. Curr Opin Struct Biol. 2007;17:513-20.

36. Akahani S, Nangia-Makker P, Inohara H, Kim HR, Raz A. Galectin-3: a novel antiapoptotic molecule with a functional BH1 (NWGR) domain of Bcl-2 family. Cancer Res. 1997;57:5272-6.

37. Lavrik IN, Eils R, Fricker N, Pforr C, Krammer PH. Understanding apoptosis by systems biology approaches. Mol Biosyst. 2009;5:1105-11.

38. Scaffidi C, Medema JP, Krammer PH, Peter ME. FLICE is predominantly expressed as two functionally active isoforms, caspase-8/a and caspase-8/b. J Biol Chem. 1997;272:26953-8.

39. Lavrik I, Krueger A, Schmitz I, Baumann S, Weyd H, Krammer $\mathrm{PH}$, et al. The active caspase- 8 heterotetramer is formed at the CD95 DISC. Cell Death Differ. 2003;10:144-5.

40. Korsmeyer SJ, Wei MC, Saito M, Weiler S, Oh KJ, Schlesinger PH. Pro-apoptotic cascade activates BID, which oligomerizes BAK or BAX into pores that result in the release of cytochrome C. Cell Death Differ. 2000;7:1166-73.

41. Keppler OT, Peter ME, Hinderlich S, Moldenhauer G, Stehling P, Schmitz I, et al. Differential sialylation of cell surface glycoconjugates in a human B lymphoma cell line regulates susceptibility for CD95 (APO-1/Fas)-mediated apoptosis and for infection by a lymphotropic virus. Glycobiology. 1999;9:557-69.

42. Shatnyeva OM, Kubarenko AV, Weber CE, Pappa A, SchwartzAlbiez R, Weber AN, et al. Modulation of the CD95-induced apoptosis: the role of CD95 N-glycosylation. PLoS ONE. 2011;6:e19927.

43. Swindall AF, Bellis SL. Sialylation of the Fas death receptor by ST6Gal-I provides protection against Fas-mediated apoptosis in colon carcinoma cells. J Biol Chem. 2011;286:22982-90.

44. Lichtenstein RG, Rabinovich GA. Glycobiology of cell death: when glycans and lectins govern cell fate. Cell Death Differ. 2013;20:976-86.
45. Charlier E, Conde C, Zhang J, Deneubourg L, Di Valentin E, Rahmouni S, et al. SHIP-1 inhibits CD95/APO-1/Fas-induced apoptosis in primary $\mathrm{T}$ lymphocytes and $\mathrm{T}$ leukemic cells by promoting CD95 glycosylation independently of its phosphatase activity. Leukemia. 2010;24:821-32.

46. Feig C, Tchikov V, Schutze S, Peter ME. Palmitoylation of CD95 facilitates formation of SDS-stable receptor aggregates that initiate apoptosis signaling. EMBO J. 2007;26:221-31.

47. Peter ME, Hellbardt S, Schwartz-Albiez R, Westendorp MO, Walczak H, Moldenhauer G, et al. Cell surface sialylation plays a role in modulating sensitivity towards APO-1-mediated apoptotic cell death. Cell Death Differ. 1995;2:163-71.

48. Li Y, Yang X, Nguyen AH, Brockhausen I. Requirement of Nglycosylation for the secretion of recombinant extracellular domain of human Fas in HeLa cells. Int J Biochem Cell Biol. 2007;39:1625-36.

49. Dufour F, Rattier T, Shirley S, Picarda G, Constantinescu AA, Morle A, et al. N-glycosylation of mouse TRAIL-R and human TRAIL-R1 enhances TRAIL-induced death. Cell Death Differ. 2017;24:500-10.

50. Wagner KW, Punnoose EA, Januario T, Lawrence DA, Pitti RM, Lancaster K, et al. Death-receptor O-glycosylation controls tumor-cell sensitivity to the proapoptotic ligand Apo2L/TRAIL. Nat Med. 2007;13:1070-7.

51. Estornes Y, Dondelinger Y, Weber K, Bruggeman I, Peall A, MacFarlane $M$, et al. N-glycosylation of mouse TRAIL-R restrains TRAIL-induced apoptosis. Cell Death Dis. 2018;9:494.

52. Cullen SP, Henry CM, Kearney CJ, Logue SE, Feoktistova M, Tynan GA, et al. Fas/CD95-induced chemokines can serve as "find-me" signals for apoptotic cells. Mol Cell. 2013;49:1034-48.

53. Mazurek N, Byrd JC, Sun Y, Hafley M, Ramirez K, Burks J, et al. Cell-surface galectin-3 confers resistance to TRAIL by impeding trafficking of death receptors in metastatic colon adenocarcinoma cells. Cell Death Differ. 2012;19:523-33.

54. Kobayashi T, Shimura T, Yajima T, Kubo N, Araki K, Wada W, et al. Transient silencing of galectin-3 expression promotes both in vitro and in vivo drug-induced apoptosis of human pancreatic carcinoma cells. Clin Exp Metastas-. 2011;28:367-76.

55. Nangia-Makker P, Hogan V, Raz A. Galectin-3 and cancer stemness. Glycobiology. 2018;28:172-81.

56. Fukumori T, Takenaka Y, Oka N, Yoshii T, Hogan V, Inohara $\mathrm{H}$, et al. Endogenous galectin-3 determines the routing of CD95 apoptotic signaling pathways. Cancer Res. 2004;64:3376-9.

57. Brandt B, Buchse T, Abou-Eladab EF, Tiedge M, Krause E, Jeschke U, et al. Galectin-1 induced activation of the apoptotic death-receptor pathway in human Jurkat $\mathrm{T}$ lymphocytes. Histochem Cell Biol. 2008;129:599-609.

58. Matarrese P, Tinari A, Mormone E, Bianco GA, Toscano MA, Ascione B, et al. Galectin-1 sensitizes resting human T lymphocytes to Fas (CD95)-mediated cell death via mitochondrial hyperpolarization, budding, and fission. J Biol Chem. 2005;280: 6969-85.

59. Yang R, Hung MC. The role of T-cell immunoglobulin mucin-3 and its ligand galectin-9 in antitumor immunity and cancer immunotherapy. Sci China Life Sci. 2017;60:1058-64.

60. Fujita K, Iwama H, Oura K, Tadokoro T, Samukawa E, Sakamoto T, et al. Cancer therapy due to apoptosis: Galectin-9. Int J Mol Sci. 2017;18:E74.

61. Kobayashi T, Kuroda J, Ashihara E, Oomizu S, Terui Y, Taniyama A, et al. Galectin-9 exhibits anti-myeloma activity through JNK and p38 MAP kinase pathways. Leukemia. 2010;24:843-50.

62. Kuroda J, Yamamoto M, Nagoshi H, Kobayashi T, Sasaki N, Shimura Y, et al. Targeting activating transcription factor 3 by Galectin-9 induces apoptosis and overcomes various types of 
treatment resistance in chronic myelogenous leukemia. Mol Cancer Res. 2010;8:994-1001.

63. Okudaira T, Hirashima M, Ishikawa C, Makishi S, Tomita M, Matsuda T, et al. A modified version of galectin-9 suppresses cell growth and induces apoptosis of human $\mathrm{T}$-cell leukemia virus type I-infected T-cell lines. Int J Cancer. 2007;120:2251-61.

64. Wiersma VR, de Bruyn M, Helfrich W, Bremer E. Therapeutic potential of Galectin-9 in human disease. Med Res Rev. 2013;33 (Suppl 1):E102-26.

65. Bremer E, van Dam G, Kroesen BJ, de Leij L, Helfrich W. Targeted induction of apoptosis for cancer therapy: current progress and prospects. Trends Mol Med. 2006;12:382-93.

66. Mallucci L, Wells V, Danikas A, Davies D. Turning cell cycle controller genes into cancer drugs. A role for an antiproliferative cytokine (betaGBP). Biochem Pharmacol. 2003; 66:1563-9.

67. Hahn HP, Pang M, He J, Hernandez JD, Yang RY, Li LY, et al. Galectin-1 induces nuclear translocation of endonuclease $\mathrm{G}$ in caspase- and cytochrome C-independent $\mathrm{T}$ cell death. Cell Death Differ. 2004;11:1277-86.

68. Adams L, Scott GK, Weinberg CS. Biphasic modulation of cell growth by recombinant human galectin-1. Biochim Biophys Acta. 1996;1312:137-44

69. Belanis L, Plowman SJ, Rotblat B, Hancock JF, Kloog Y. Galectin-1 is a novel structural component and a major regulator of h-ras nanoclusters. Mol Biol Cell. 2008;19:1404-14.

70. Michael JV, Goldfinger LE. Concepts and advances in cancer therapeutic vulnerabilities in RAS membrane targeting. Semin Cancer Biol. 2017. https://doi.org/10.1016/j.semcancer.2017. 11.021.

71. Sturm A, Lensch M, Andre S, Kaltner H, Wiedenmann B, Rosewicz $\mathrm{S}$, et al. Human galectin-2: novel inducer of $\mathrm{T}$ cell apoptosis with distinct profile of caspase activation. J Immunol. 2004;173:3825-37.

72. Stillman BN, Hsu DK, Pang M, Brewer CF, Johnson P, Liu FT, et al. Galectin-3 and galectin-1 bind distinct cell surface glycoprotein receptors to induce $\mathrm{T}$ cell death. $\mathrm{J}$ Immunol. 2006;176:778-89.

73. Hsu DK, Chen HY, Liu FT. Galectin-3 regulates T-cell functions. Immunol Rev. 2009;230:114-27.

74. Stupack DG, Puente XS, Boutsaboualoy S, Storgard CM, Cheresh DA. Apoptosis of adherent cells by recruitment of caspase-8 to unligated integrins. J Cell Biol. 2001;155:459-70.

75. Furtak V, Hatcher F, Ochieng J. Galectin-3 mediates the endocytosis of beta-1 integrins by breast carcinoma cells. Biochem Biophys Res Commun. 2001;289:845-50.

76. Garner OB, Baum LG. Galectin-glycan lattices regulate cellsurface glycoprotein organization and signalling. Biochem Soc Trans. 2008;36(Pt 6):1472-7.

77. Hanada M, Aime-Sempe C, Sato T, Reed JC. Structure-function analysis of $\mathrm{Bcl}-2$ protein. Identification of conserved domains important for homodimerization with $\mathrm{Bcl}-2$ and heterodimerization with Bax. J Biol Chem. 1995;270:11962-9.

78. Hsu DK, Zuberi RI, Liu FT. Biochemical and biophysical characterization of human recombinant IgE-binding protein, an S-type animal lectin. J Biol Chem. 1992;267:14167-74.

79. Harazono Y, Nakajima K, Raz A. Why anti-Bcl-2 clinical trials fail: a solution. Cancer Metastasis Rev. 2014;33:285-94.

80. Oka N, Nakahara S, Takenaka Y, Fukumori T, Hogan V, Kanayama HO, et al. Galectin-3 inhibits tumor necrosis factorrelated apoptosis-inducing ligand-induced apoptosis by activating Akt in human bladder carcinoma cells. Cancer Res. 2005; 65:7546-53.

81. Wang Y, Nangia-Makker P, Balan V, Hogan V, Raz A. Calpain activation through galectin-3 inhibition sensitizes prostate cancer cells to cisplatin treatment. Cell Death Dis. 2010;1:e101.
82. Cecchinelli B, Lavra L, Rinaldo C, Iacovelli S, Gurtner A, Gasbarri A, et al. Repression of the antiapoptotic molecule galectin-3 by homeodomain-interacting protein kinase 2activated p53 is required for p53-induced apoptosis. Mol Cell Biol. 2006;26:4746-57.

83. Takenaka Y, Fukumori T, Yoshii T, Oka N, Inohara H, Kim HR, et al. Nuclear export of phosphorylated galectin-3 regulates its antiapoptotic activity in response to chemotherapeutic drugs. Mol Cell Biol. 2004;24:4395-406.

84. Yu F, Finley RL Jr, Raz A, Kim HR. Galectin-3 translocates to the perinuclear membranes and inhibits cytochrome $\mathrm{C}$ release from the mitochondria. A role for synexin in galectin-3 translocation. J Biol Chem. 2002;277:15819-27.

85. Villeneuve C, Baricault L, Canelle L, Barboule N, Racca C, Monsarrat B, et al. Mitochondrial proteomic approach reveals galectin-7 as a novel BCL-2 binding protein in human cells. Mol Biol Cell. 2011;22:999-1013.

86. Kuwabara I, Kuwabara Y, Yang RY, Schuler M, Green DR, Zuraw BL, et al. Galectin-7 (PIG1) exhibits pro-apoptotic function through JNK activation and mitochondrial cytochrome C release. J Biol Chem. 2002;277:3487-97.

87. Eshkar Sebban L, Ronen D, Levartovsky D, Elkayam O, Caspi D, Aamar S, et al. The involvement of CD44 and its novel ligand galectin-8 in apoptotic regulation of autoimmune inflammation. $\mathbf{J}$ Immunol. 2007;179:1225-35.

88. Norambuena A, Metz C, Vicuna L, Silva A, Pardo E, Oyanadel C, et al. Galectin-8 induces apoptosis in Jurkat $\mathrm{T}$ cells by phosphatidic acid-mediated ERK1/2 activation supported by protein kinase A down-regulation. J Biol Chem. 2009;284:12670-9.

89. Kashio Y, Nakamura K, Abedin MJ, Seki M, Nishi N, Yoshida $\mathrm{N}$, et al. Galectin-9 induces apoptosis through the calciumcalpain-caspase-1 pathway. J Immunol. 2003;170:3631-6.

90. Wada J, Ota K, Kumar A, Wallner EI, Kanwar YS. Developmental regulation, expression, and apoptotic potential of galectin-9, a beta-galactoside binding lectin. J Clin Invest. 1997;99:2452-61.

91. Hotta K, Funahashi T, Matsukawa Y, Takahashi M, Nishizawa $\mathrm{H}$, Kishida K, et al. Galectin-12, an adipose-expressed galectinlike molecule possessing apoptosis-inducing activity. J Biol Chem. 2001;276:34089-97.

92. El Leithy AA, Helwa R, Assem MM, Hassan NH. Expression profiling of cancer-related galectins in acute myeloid leukemia. Tumour Biol. 2015;36:7929-39.

93. Tejler J, Leffler H, Nilsson UJ. Synthesis of O-galactosyl aldoximes as potent LacNAc-mimetic galectin-3 inhibitors. Bioorg Med Chem Lett. 2005;15:2343-5.

94. Salameh BA, Leffler H, Nilsson UJ. 3-(1,2,3-Triazol-1-yl)-1-thiogalactosides as small, efficient, and hydrolytically stable inhibitors of galectin-3. Bioorg Med Chem Lett. 2005;15:3344-6.

95. Cumpstey I, Carlsson S, Leffler H, Nilsson UJ. Synthesis of a phenyl thio-beta-D-galactopyranoside library from 1,5-difluoro2,4-dinitrobenzene: discovery of efficient and selective monosaccharide inhibitors of galectin-7. Org Biomol Chem. 2005; 3:1922-32.

96. Thijssen VL, Postel R, Brandwijk RJ, Dings RP, Nesmelova I, Satijn S, et al. Galectin-1 is essential in tumor angiogenesis and is a target for antiangiogenesis therapy. Proc Natl Acad Sci USA. 2006;103:15975-80.

97. Wdowiak K, Francuz T, Gallego-Colon E, Ruiz-Agamez N, Kubeczko M, Grochola I, et al. Galectin targeted therapy in oncology: current knowledge and perspectives. Int $\mathrm{J}$ Mol Sci. 2018;19:E210.

98. Hsieh SH, Ying NW, Wu MH, Chiang WF, Hsu CL, Wong TY, et al. Galectin-1, a novel ligand of neuropilin-1, activates VEGFR-2 signaling and modulates the migration of vascular endothelial cells. Oncogene. 2008;27:3746-53. 
99. Zucchetti M, Bonezzi K, Frapolli R, Sala F, Borsotti P, Zangarini $\mathrm{M}$, et al. Pharmacokinetics and antineoplastic activity of galectin1-targeting OTX008 in combination with sunitinib. Cancer Chemother Pharmacol. 2013;72:879-87.

100. Kindt N, Journe F, Ghanem GE, Saussez S. Galectins and carcinogenesis: their role in head and neck carcinomas and thyroid carcinomas. Int J Mol Sci. 2017;18:E2745.
101. Michael JV, Wurtzel JG, Goldfinger LE. Inhibition of Galectin-1 sensitizes HRAS-driven tumor growth to rapamycin treatment. Anticancer Res. 2016;36:5053-61.

102. Streetly MJ, Maharaj L, Joel S, Schey SA, Gribben JG, Cotter FE. GCS-100, a novel galectin-3 antagonist, modulates MCL-1, NOXA, and cell cycle to induce myeloma cell death. Blood. 2010;115:3939-48. 